\title{
Two-Dimensional Spectroscopy of Chlorophyll $a$ Excited-State Equilibration in Light-Harvesting Complex II
}

Parveen Akhtar, ${ }^{\dagger, t}$ Cheng Zhang, ${ }^{\dagger}$ Thanh Nhut Do, ${ }^{\dagger}$ Gyözö Garab, ${ }^{+}$Petar H. Lambrev, *t,t+ HoweSiang Tan *广

† School of Physical and Mathematical Sciences, Nanyang Technological University, 21 Nanyang Link, Singapore 637371

* Biological Research Centre, Hungarian Academy of Sciences, Temesvári krt. 62, Szeged 6726, Hungary

\section{AUTHOR INFORMATION}

\section{Corresponding authors}

Howe-Siang Tan (HoweSiang@ntu.edu.sg), Petar H. Lambrev (lambrev.petar@brc.mta.hu)

ABSTRACT. Excited-state relaxation dynamics and energy-transfer processes in the chlorophyll $a$ (Chl $a$ ) manifold of the light-harvesting complex II (LHCII) were examined at physiological temperature using femtosecond two-dimensional electronic spectroscopy (2DES). The experiments were done under conditions free from singlet-singlet annihilation and anisotropic decay. Energy transfer between the different domains of the $\mathrm{Chl} a$ manifold was found to proceed on timescales from hundreds of femtoseconds to five picoseconds, before reaching equilibration. No component slower than 10 ps was observed in the spectral equilibration dynamics. We clearly observe the bidirectional (uphill and downhill) energy transfer of the equilibration process between excited states. This bidirectional energy flow, although implicit in the modelling and simulation of the EET processes, has not been observed in any prior transient absorption studies. Furthermore, we identified the spectral forms associated with the different energy transfer lifetimes in the equilibration process. 


\section{TOC GRAPHICS}

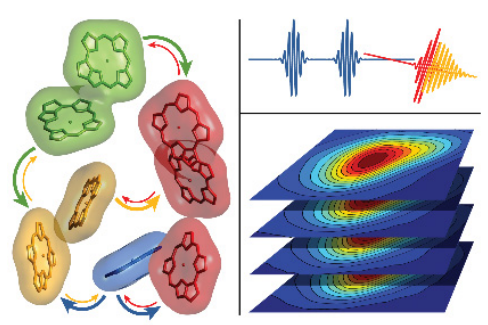

KEYWORDS. excitation energy transfer, light harvesting, multidimensional spectroscopy, photosynthesis, pigment-protein complexes, time-resolved spectroscopy 
All photosynthetic organisms rely on light-harvesting antenna systems to maximize the flux of excitation energy at the photochemical reaction centers. Efficient photochemistry relies on the fact that the excitation energy transfer (EET) through the exciton network occurs on a much shorter timescale than the excitation lifetime. Light-harvesting complex II, the most abundant pigmentprotein complex in the biosphere, ${ }^{1,2}$ is the major light-harvesting antenna complex in plants and green algae. The relatively rigid protein structure of $\mathrm{LHCII}^{3,4}$ ensures fine-tuned localization and environment of the noncovalently bound pigment molecules - chlorophyll (Chl) $a$ and $b$ and xanthophylls. The EET dynamics in LHCII has been studied extensively over the past decades using various time-resolved spectroscopic techniques and modelling approaches. ${ }^{1}$ Yet, despite the wealth of literature data, there is no complete consensus on the dynamics and pathways of EET, particularly in the equilibration process in the manifold of $\mathrm{Chl} a$ exciton states. Upon photoexcitation, due to the narrow spread in energy of the manifold of Chl $a$ exciton states, significant uphill and downhill EET will occur concurrently, leading eventually to a dynamic thermal equilibrium. A wide range of lifetimes for this equilibration process have been reported for LHCII monomers and trimers. At temperatures of $77 \mathrm{~K}$ or lower, excitation on the blue side of the $Q_{y}$ band of Chl $a$ is followed by downhill EET with time constants from hundreds of femtoseconds to about 20 ps. $^{5-8}$ At physiological temperatures, 5-7 ps lifetime components assigned to Chl a exciton equilibration have been observed by transient absorption ${ }^{9-11}$, fluorescence $^{12}$ and three-pulse photon echo. ${ }^{13,14}$ However, significantly longer lifetimes, in the range of tens of picoseconds and more, have also been reported. ${ }^{9,15,16}$ The slowest lifetimes have been assigned to one Chl $a$ pigment absorbing in the region of $665-670 \mathrm{~nm} .^{7,17}$ This has been referred to as the bottleneck state and, in agreement with structure-based modelling, assigned to Chl $a 604^{17,18}$ in the crystal structure of Liu et al. ${ }^{19}$ The assignment has been challenged by more recent modelling ${ }^{20}$ that incidentally also predicts slow $\mathrm{Chl} a-\mathrm{Chl} a$ equilibration lifetimes in the tens of picoseconds region. ${ }^{21}$ Thus, at present neither the timescales nor the molecular pathways of exciton equilibration in the $\mathrm{Chl} a$ domain are unambiguously established.

Two-dimensional coherent electronic spectroscopy (2DES) has advantages over conventional pump-probe by providing separate spectral information of donor and acceptor molecules participating in EET. ${ }^{22,23}$ 2DES has been used to examine kinetics and pathways of EET from Chl $b$ to Chl $a$ in isolated, detergent-solubilized LHCII at low temperature ${ }^{17,24}$ and recently at physiological temperature. ${ }^{25-28}$ In order to clearly observe such dynamics, purely absorptive 2D 
spectra need to be obtained and analyzed without compromising the spectral resolution and precision, time range, or dynamic range (signal-to-noise ratio). There are several sources of artefacts that complicate the analysis. One source may be inaccurate 'phasing', typically associated with 2DES based on the boxcar geometry approach, where two separate sets of data (rephasing and non-rephasing) are summed to generate the purely absorptive 2D spectra. Slight inaccuracy in determining the relative time delays of these two sets of data will produce distortions to the $2 \mathrm{D}$ peak shapes that may then be falsely assigned to non-existing processes. ${ }^{29}$ Other potential artefacts arise from multiphoton processes, primarily singlet-singlet annihilation, which occurs when multiple excitations are present in the same LHCII complex ${ }^{30}$ and can seriously distort the excitedstate kinetics.

With the above limitations in mind, we performed 2DES experiments aiming to resolve the room-temperature dynamics of exciton equilibration in the $\mathrm{Chl} a$ manifold in LHCII trimers, with excitation pulses centered at $675 \mathrm{~nm}$, covering the Chl $a$ absorption band (Supporting Figure S1). One major advantage of our current 2DES technique using a pulse-shaper assisted pump-probe geometry as compared to the more conventional boxcar geometry is that the measured 2D spectra are automatically purely absorptive. ${ }^{31,32}$ No phasing is therefore necessary and the ambiguity of whether the peak shapes are distorted is not an issue. To avoid singlet-singlet annihilation, we used excitation pulse energies low enough to virtually eliminate the possibility for multiple simultaneous excitations in the complexes. In addition, the first two excitation pulses were planepolarized at the magic angle relative to the third interaction pulses to measure only the population dynamics of the system. The data are also of sufficiently high dynamic range to perform global multicomponent kinetic analysis to obtain quantitative values for the energy equilibration dynamics. 

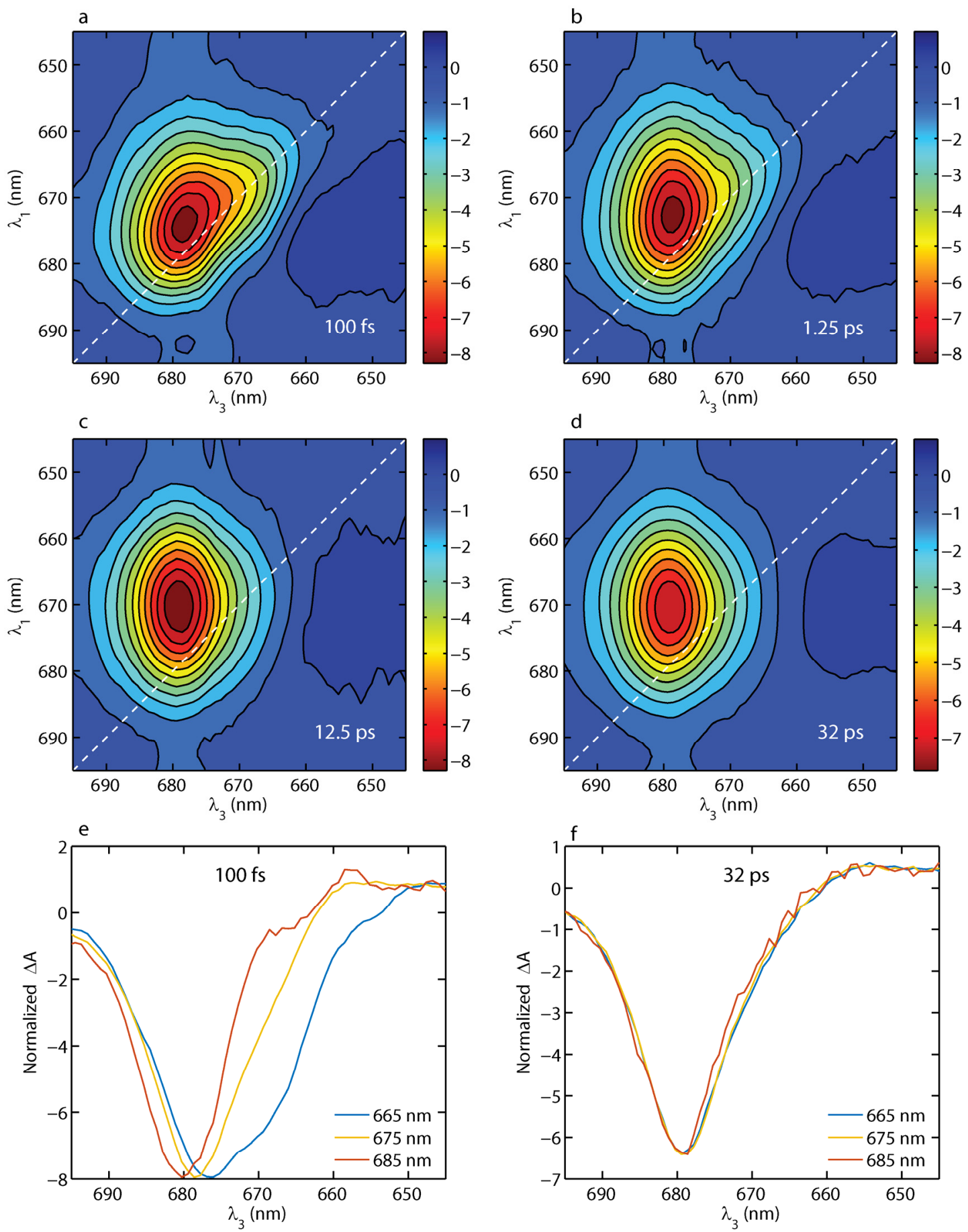

Figure 1. Purely absorptive 2D spectra of LHCII trimers recorded in the Chl $Q_{y}$ region with excitation pulses at 675 $\mathrm{nm}$. a, $\mathrm{d}$ - contour plots of the 2D spectra recorded at the indicated waiting times $\left(T_{w}\right)$, plotted in terms of excitation wavelength $\lambda_{1}$ and detection wavelength $\lambda_{3}$. Contour lines mark areas of equal absorptive signal intensity given in the color scale bars. e, f-horizontal slices at excitation wavelengths of 665,675 and $685 \mathrm{~nm}$ extracted from the 2D spectra at waiting times $0.1 \mathrm{ps}$ (b) and $30 \mathrm{ps}$ (d). The slices are normalized at their negative maxima for easier comparison. 
Representative 2D spectra for selected waiting times $T_{w}$ are plotted in Figure 1. A spectrum at coordinates $\left(\lambda_{1}, \lambda_{3}\right)$ reflects the conditional differential absorption detected at wavelength $\lambda_{3}$ after excitation at $\lambda_{1}$. The negative signal at detection wavelengths above $660 \mathrm{~nm}$ is contributed mainly by ground state bleaching (GSB) and stimulated emission (SE) from the first singlet excited state of Chl $a$. The appearance of cross peaks in the 2D spectra mainly reflects redistribution of excitation energy (EET) during the waiting time $T_{w}$. Because of preferentially downhill EET, the signal gradually red shifts. If the system of coupled chromophores has reached a thermally equilibrated state, the signal becomes independent of excitation wavelength (Figure 1f). Thus, we can conclude that, firstly, part of the excitations from higher-energy $\mathrm{Chl} a$ are transferred to lowerenergy states within 150 fs (Figure 1a) and, secondly, by noticing that there is no change between 2D spectra at $T_{w}=12.5$ and $T_{w}=32 \mathrm{ps}$ (Figure 1c, d, respectively), equilibration among all Chl $a$ 's is complete after about $10 \mathrm{ps}$.
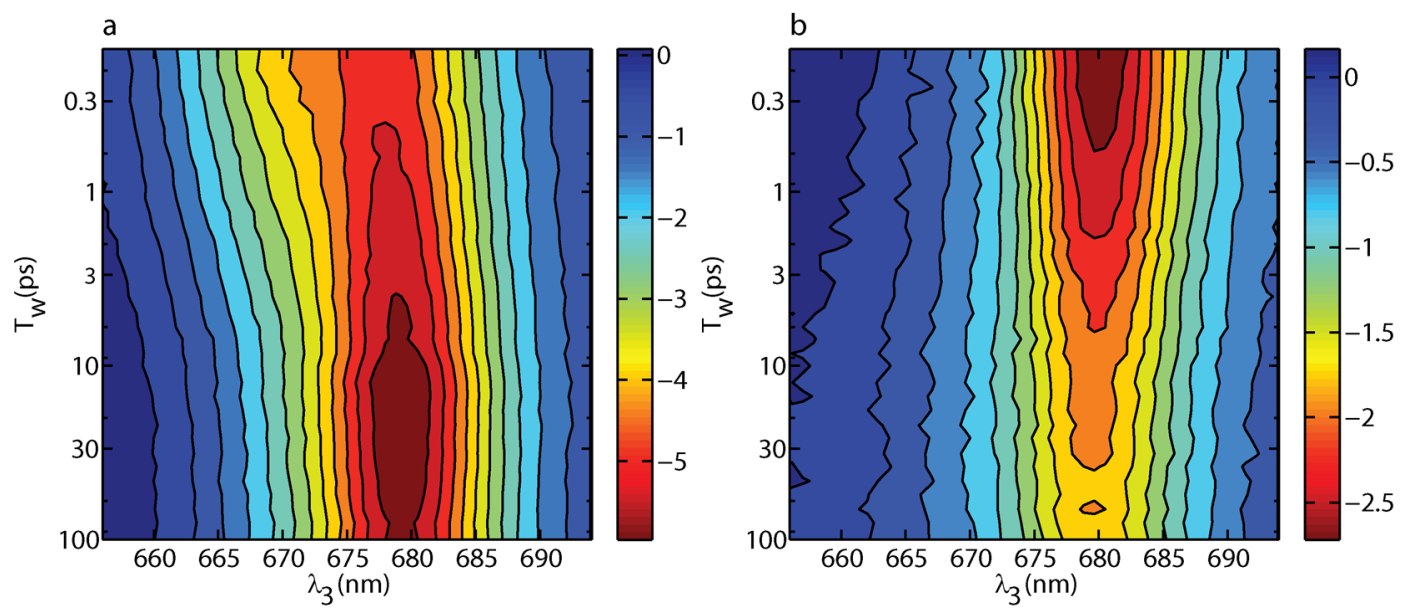

Figure 2. Contour plots showing the waiting-time dependence of the LHCII $2 \mathrm{D}$ signal at two fixed excitation wavelengths $\lambda_{1}=665 \mathrm{~nm}$ (a) and $685 \mathrm{~nm}$ (b). The plots can be understood as analogous to pump-probe experiments with pump wavelengths, corresponding to $\lambda_{1}$ - i.e. on the blue and red side of the $Q_{y}$ absorption band of LHCII.

At physiological temperatures, the energy gaps between several exciton states in LHCII are within the thermal energy (the Boltzmann term $k T$ spans about $10 \mathrm{~nm}$ in this region); for the equilibration process, both forward (downhill) and reverse (uphill) EET are expected to occur. ${ }^{13,28}$ This is clearly seen in Figure 2, which shows the $T_{w}$ dependence of the 2D signal amplitude at the high-energy $(665 \mathrm{~nm})$ and low-energy side $(685 \mathrm{~nm})$ of the $Q_{y}$ absorption band. In Figure 2a (Figure 2b) we see the spectrum peak gradually red shifts (blue shifts) with increasing $T_{w}$ due to downhill (uphill) EET. The peak position in both Figure 2a,b converges to $679 \mathrm{~nm}$ by $~ 10 \mathrm{ps}$. 

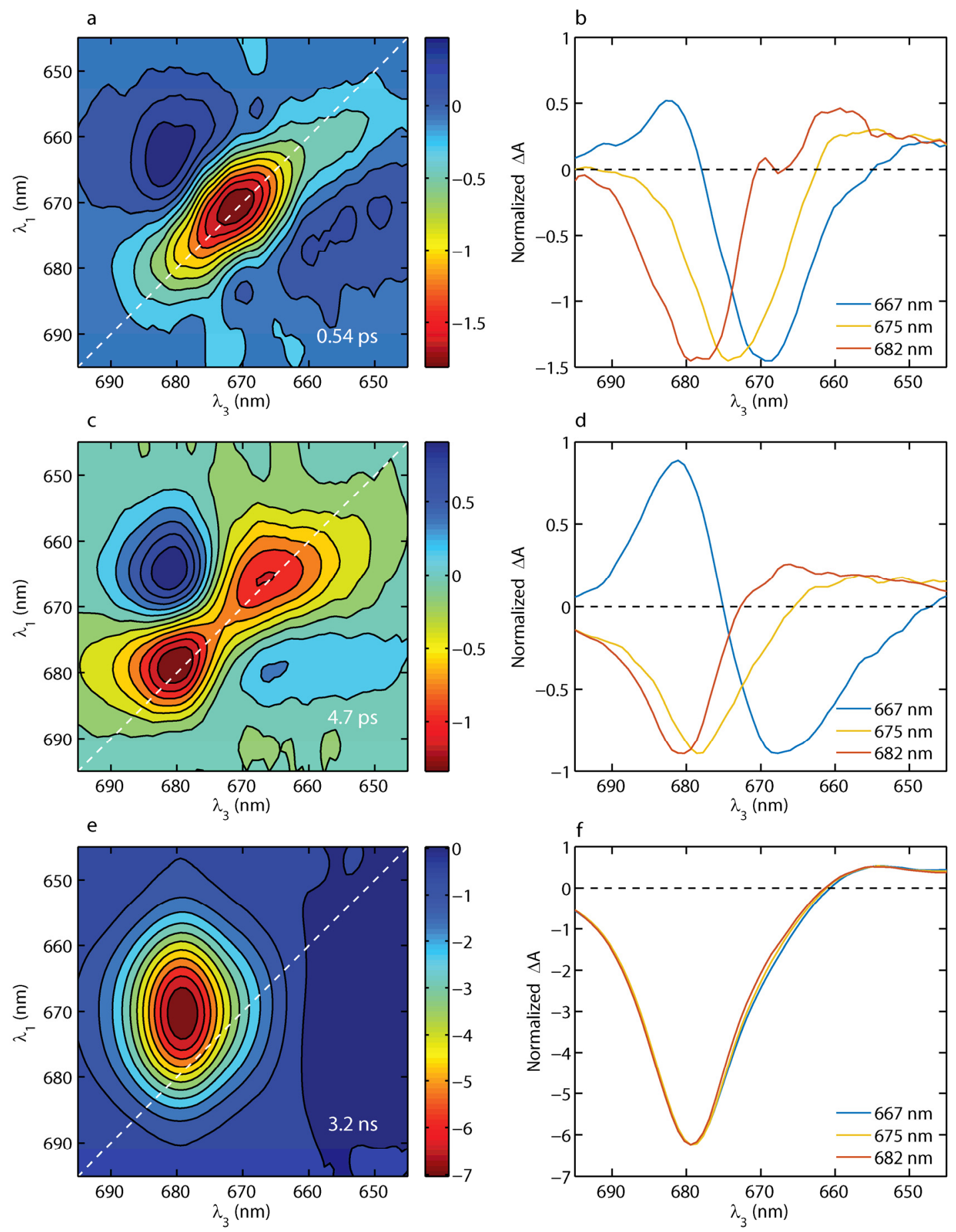

Figure 3. 2D decay-associated spectra (2D DAS) of LHCII trimers, resulting from a three-exponential fit of the transient 2D signals from 150 fs to 64 ps with best-fit lifetimes of $0.54 \mathrm{ps}, 4.7 \mathrm{ps}$, and $3.2 \mathrm{~ns}$. a, c, d - contour plots of the 2D DAS (amplitude in the color scale bars). b, e, $\mathrm{f}$ - horizontal slices of the corresponding 2D DAS at three excitation wavelengths, normalized at their negative maxima. 
Furthermore, following the overall signal amplitude as the spectral evolution progresses (Figure 2), it can also be concluded that low-energy $\mathrm{Chl} a$ states (at detection wavelengths near $680 \mathrm{~nm}$ ) have larger dipole strength, i.e. they are superradiant. This is likely because the states are delocalized over excitonically coupled pigments with favorably oriented transition dipole moments. ${ }^{33}$ The observation is in agreement with the nonlinear polarized fluorescence study of Schubert et al. ${ }^{34}$ who estimated that the emitting state in LHCII is superradiant, having 2-fold larger dipole strength compared to monomeric $\mathrm{Chl}$.

We performed global analysis (see Supporting Methods) on the 2D spectra at $T_{w}$ from 100 fs to $300 \mathrm{ps}$ to obtain a quantitative estimation of the spectral equilibration kinetics. Three 2D decayassociated spectra (2D DAS) with lifetimes $0.54 \mathrm{ps}, 4.7 \mathrm{ps}$, and $3.2 \mathrm{~ns}$ were necessary and sufficient to obtain a good fit (Figure 3). The errors in the first two lifetimes did not exceed 30\% in repeated experiments, but were larger (up to $160 \%$ ) for the final lifetime as it is beyond the measurement range.

Negative and positive amplitudes indicate decay and rise of GSB/SE signal, respectively. Positive amplitudes can also represent decay of excited-state absorption (ESA). EET is identified as a negative diagonal peak $\left(\lambda_{1}=\lambda_{3}\right)$ at the absorption wavelength of the donor spectral form and a positive peak at $\lambda_{1}$ matching the donor and $\lambda_{3}$ matching the acceptor. For reversible EET in the equilibration dynamics, two antisymmetric positive/negative pairs are observed in the 2D DAS. The downhill (uphill) EET produces a cross-peak above (below) the diagonal, at $\lambda_{3}>\lambda_{1}\left(\lambda_{3}<\lambda_{1}\right)$. In a recent paper Duan et al. ${ }^{28}$ have reported such above-/below-diagonal 2D DAS cross-peaks, which they attributed to EET between Chl $a$ and $b$ occurring on a timescale of $1.1 \mathrm{ps}$. We note that, although the forward and reverse EET rates may be different, depending on the energy gap and the size of the pools, the observable spectral changes always occur with the same equilibration rate (timescale). This relationship makes the 2D DAS a powerful means to identify EET pathways. Here, antisymmetrically positioned positive/negative peaks are evident in the first two 2D DAS (Figure 3); the latter one (4.7 ps) shows reversible EET between $667 \mathrm{~nm}$ and $680 \mathrm{~nm}$ spectral forms. The shape of this 2D DAS is reminiscent of the $1.1 \mathrm{ps} 2 \mathrm{D}$ DAS reported by Duan et al. ${ }^{28}$ The amplitude ratio of the $667 \rightarrow 680 \mathrm{~nm}$ and the $680 \rightarrow 667 \mathrm{~nm}$ cross-peaks, after adjustment for the excitation pulse spectrum, reflects the forward/reverse rate constant ratio. ${ }^{28}$ Thus, we have clearly resolved and characterized the bidirectional thermodynamic equilibration between $\mathrm{Chl} a$ excited states coupled by reversible EET. Whereas the 4.7 ps 2D DAS appears to involve a single 
pathway between two Chl pools, the 0.54 ps component features more than one EET pathway with a major contribution from spectral forms lying in the middle of the Chl $a$ region $(672-675 \mathrm{~nm})$. This faster equilibration component was not observed by Duan et al. ${ }^{28}$ The final 2D DAS (3.2 ns) does not describe any EET - the spectral shape is the same at any excitation wavelength and features a main negative GSB/SE peak and a small valued positive band at $\lambda_{3}<660 \mathrm{~nm}$ attributed to ESA. The DAS represents decay of the completely equilibrated state and is consistent with the documented excited-state lifetime of LHCII. ${ }^{35,36}$

While the measured lifetimes for the equilibration dynamics (0.54 ps and $4.7 \mathrm{ps})$ are in good agreement with numerous previous studies, we do not observe any slower spectral equilibration processes. We contend that lifetimes in the range of 10-20 ps observed previously, $9,16,26$ are contributed by singlet-singlet annihilation, which is often evident because of the non-conservative shape of the DAS, indicating excitation decay rather than EET, and has been acknowledged in earlier works as discussed by Connelly et al. ${ }^{11}$ Lending further support to this conclusion, we have performed experiments at different pulse energies to show the onset of annihilation contributions with increasing excitation rate (Supporting Figure S2). At higher pulse energies (10 nJ), the kinetics became non-exponential and an additional lifetime component was necessary to fit the data (17.6 ps, Supporting Figure S3). Crucially, if we normalized the 2D spectra to the total integrated intensity at every delay time, thus eliminating the decay, global analysis resulted in only two components with lifetimes and 2D DAS very similar to the annihilation-free data (cf. Figure 3 and Supporting Figure S4). Further increasing the excitation rate results in progressively faster annihilation kinetics distorting the exciton equilibration components. ${ }^{26}$

To validate the interpretation of the 2D DAS and reveal the spectral identities of the EET species, we simulated the $2 \mathrm{D}$ spectra with a phenomenological model consisting of four exciton states, or pools, connected by reversible (Boltzmann-factored) EET, as depicted in Figure 4 (a brief description of the theory is given in Supporting Methods). The kinetic system, solved analytically, has three EET lifetimes -0.46 ps, 0.58 ps and 4.8 ps. The first two lifetimes, contributed by EET steps $1 \rightarrow 2(663 \rightarrow 667 \mathrm{~nm})$ and $3 \rightarrow 4(673 \rightarrow 680 \mathrm{~nm})$, are of similar value and the kinetics can be described well by a single 0.53 ps 2D DAS (Figure 4c). The 4.8 ps lifetime (Figure 4d) is due to the slow $2 \rightarrow 4$ transfer, i.e. equilibration between the $663 / 667 \mathrm{~nm}$ pool and the $673 / 680 \mathrm{~nm}$ pool. The simulated 2D DAS bear close similarity to the experimental 2D DAS (Figure 3a,c), in terms of cross-peak positions and relative amplitudes. A model incorporating more states with structure- 
based constraints on the rates and energy levels, ${ }^{16,18,21,28}$ would be necessary to simulate finer details in the experimental data.

a
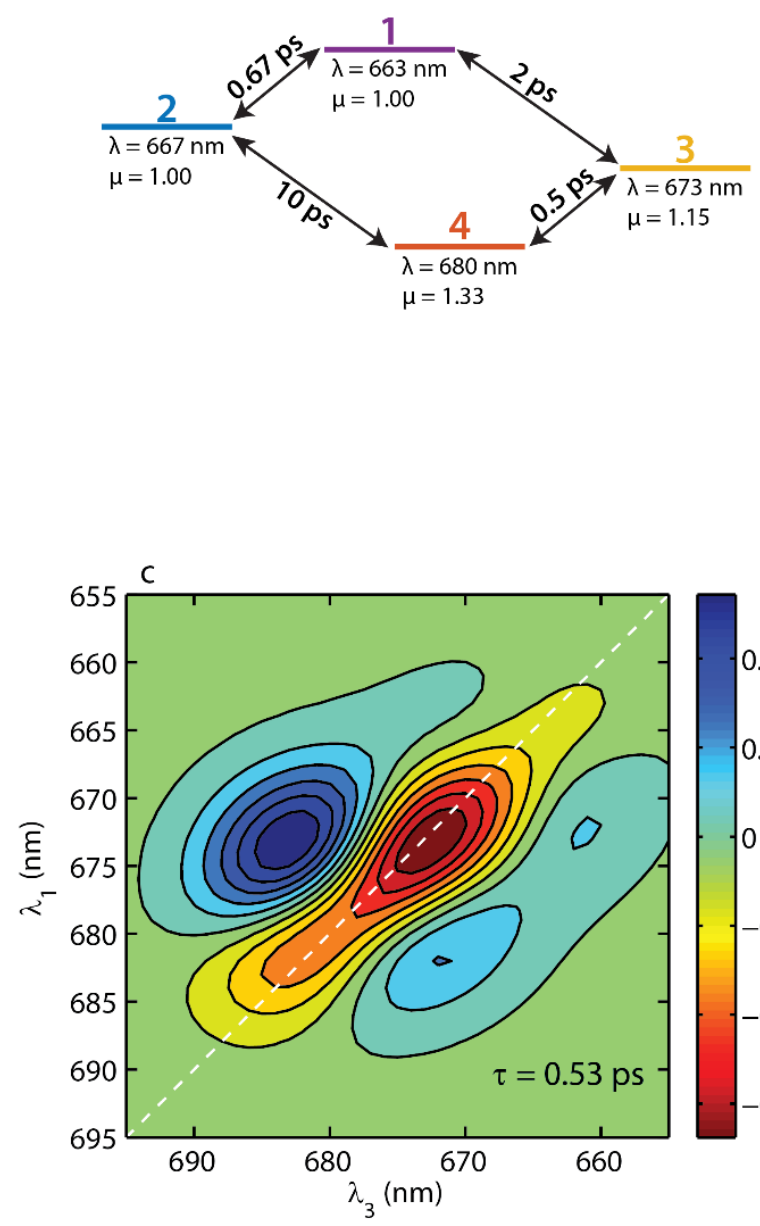
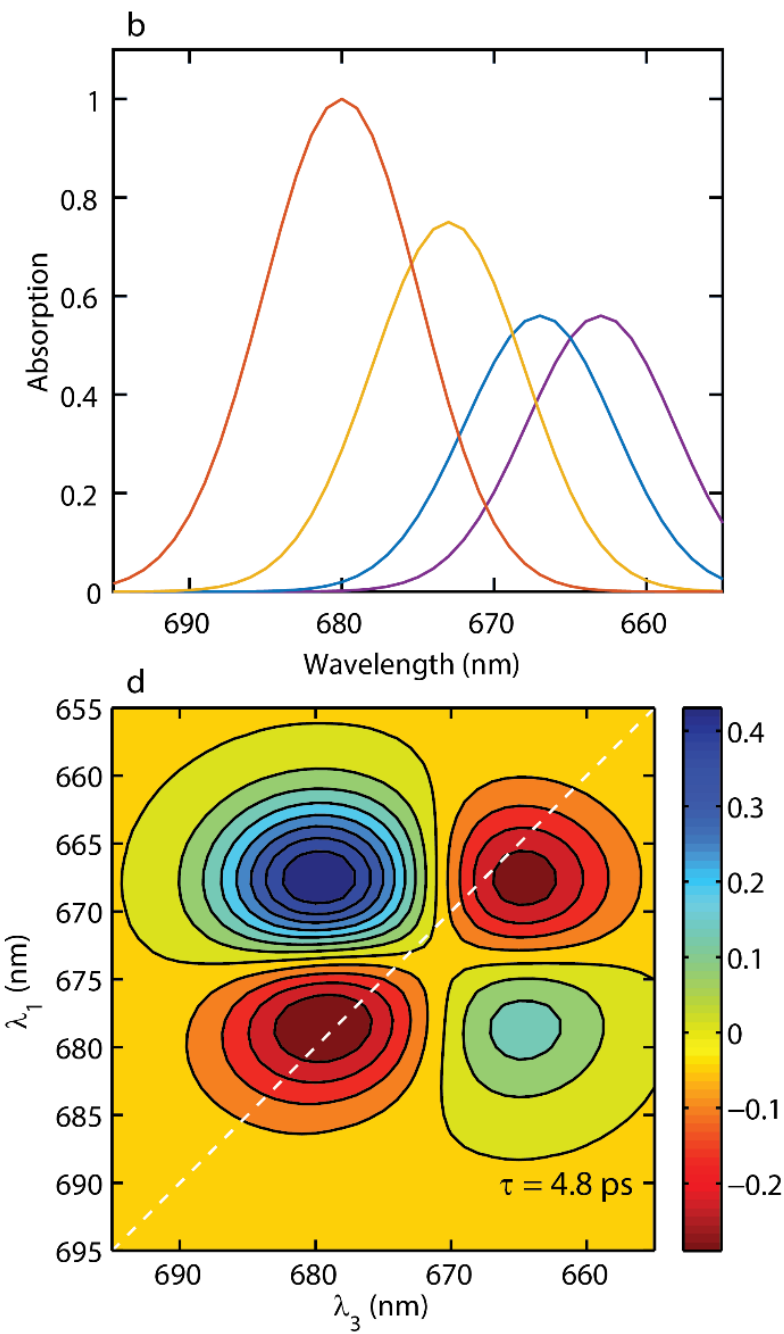

Figure 4. Theoretical model with four coupled states (Chl a), absorbing at 663, 667, 673 and $680 \mathrm{~nm}$. a- schematic diagram of the model parameters - absorption wavelength $(\lambda)$, transition dipole moment $(\mu)$, EET time constants in ps; b- simulated linear absorption spectra of the four exciton states; c, d-contour plots of 2D DAS obtained by fitting two EET lifetimes.

We can conclude that spectral equilibration in LHCII is kinetically limited by EET occurring on a 5 ps timescale between two Chl $a$ pools. The participating spectral forms $(667 \mathrm{~nm}, 680 \mathrm{~nm})$ can be unambiguously resolved in the 2D spectra, especially owing to the capability of 2DES to follow simultaneously energetically uphill and downhill EET. It is reasonable to assume, in accordance with other works, that the longer lifetime represents EET from the luminal side of the complex to 
the lower-energy Chls on the stromal side (Chl $a$ 613/614 to 610/611/612 ${ }^{19}$ ). Faster EET processes on sub-ps timescale probably represent equilibration within the luminal and stromal Chl $a$ pools. We estimate that the slow equilibration time has up to $40 \%$ contribution to the kinetics, whereas $25-35 \%$ of excitations on upper exciton states are transferred within 150 fs and $25-35 \%$ are transferred on the timescale of 500 fs (see Supporting Methods). The slower components in the range of $20 \mathrm{ps,} \mathrm{detectable} \mathrm{by} \mathrm{singlet-singlet} \mathrm{annihilation,} \mathrm{may} \mathrm{reflect} \mathrm{additional} \mathrm{spatial}$ equilibration between same-energy exciton states on different monomers in the trimer (possibly Chl $a 602 / 603^{37,38}$ ), in agreement with numerous earlier works (reviewed in ref. 2). Thus, the 2DES results presented here, while consistent with earlier data, separate the kinetics of spectral equilibration from inter-monomer transfer and, combined with new structure-based modelling, enable a more accurate and detailed understanding of the light harvesting dynamics.

\section{EXPERIMENTAL METHODS}

Trimeric LHCII samples were isolated according to the procedures given in Caffari et al. ${ }^{39}$ Briefly, PSII-enriched membrane fragments (so-called BBY) isolated from dark-adapted leaves of green-house grown pea (Pisum sativum, were solubilized with $0.7 \%$ dodecyl- $\beta$-maltoside ( $\beta$-DM)

followed by sucrose density gradient ultracentrifugation. The second band of the gradient from the top, containing LHCII trimers, was collected and washed with $10 \mathrm{mM}$ Tricine buffer (pH 7.8) and concentrated with $30 \mathrm{kDa}$ cutoff Amicon filters (Millipore), then frozen in liquid nitrogen and stored at $-80{ }^{\circ} \mathrm{C}$ until use.

For 2DES, LHCII samples were diluted in degassed buffer solution containing $20 \mathrm{mM}$ Tricine $(\mathrm{pH} 7.8)$ and $0.03 \% \beta$-DM to obtain absorption of 0.33 at the excitation wavelength $(675 \mathrm{~nm})$, in a $1 \mathrm{~mm}$ optical cell. In this regime, due to propagation effects, there will be slight broadening of the $2 \mathrm{D}$ peakshapes along the detection wavelength. ${ }^{40}$ During the $2 \mathrm{DES}$ experiments the sample was passed through a flow cell and continuously refreshed.

Fourier-transform femtosecond 2DES was performed in a partially collinear "pump-probe" geometry. ${ }^{31,41}$ The first two interaction pulses (excitation pulses) were tuned to a central wavelength of $675 \mathrm{~nm}$ with $18 \mathrm{~nm}$ bandwidth, matching the $Q_{y}$ absorption band of Chl $a$ in LHCII, 45 fs temporal width and energy of $0.5-0.8 \mathrm{~nJ}$ per pulse. An acousto-optic programmable dispersive filter pulse shaper ${ }^{42}$ (Dazzler, Fastlite) was used to scan the coherence time delay $t_{1}$ from 0 to 150 fs with 3 fs increments in a $1 \times 2$ phase-cycling scheme. ${ }^{31,41}$ White-light 
supercontinuum generated in a $2 \mathrm{~mm}$ sapphire window was used as the third interaction pulse (probe). The probe light was plane-polarized at the magic angle $\left(54.7^{\circ}\right)$ with respect to the polarization of the excitation pulses. The time delay between the second excitation pulse and the probe pulse (population waiting time $T_{w}$ ) was varied from -0.3 ps to $600 \mathrm{ps}$ in a logarithmic progression.

\section{ACKNOWLEDGMENTS}

The work was supported by grants from the Singapore Ministry of Education Academic Research Fund (Tier 2 MOE2015-T2-039 and Tier 1 RG16/15), the Hungarian Ministry for National Economy (GINOP-2.3.2-15-2016-00001 to P.H.L.) and the Hungarian National Research, Development and Innovation Office (OTKA-K 112688 to G.G.).

\section{SUPPORTING INFORMATION}

Supporting methods: calculation of excitation rate, global analysis, EET lifetime contribution, simulation of 2DES spectra (PDF)

Supporting figures: excitation and absorption spectrum; power dependence of the pump-probe signal; 2DES with $10 \mathrm{~nJ}$ pulses (PDF)

\section{REFERENCES}

(1) van Amerongen, H.; Croce, R. Structure and Function of Photosystem II Light-Harvesting Proteins (Lhcb) of Higher Plants. In Primary Processes of Photosynthesis, Part 1; Royal Society of Chemistry: Cambridge, UK, 2007; pp 329-367.

(2) van Amerongen, H.; Croce, R. Light Harvesting in Photosystem II. Photosynth. Res. 2013, 116, $251-263$.

(3) Standfuss, J.; van Scheltinga, A. C. T.; Lamborghini, M.; Kühlbrandt, W. Mechanisms of Photoprotection and

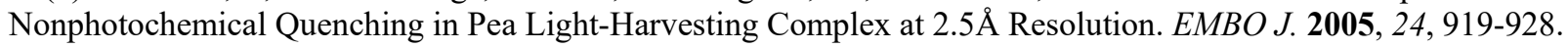

(4) Dockter, C.; Müller, A. H.; Dietz, C.; Volkov, A.; Polyhach, Y.; Jeschke, G.; Paulsen, H. Rigid Core and Flexible Terminus: Structure of Solubilized Light-Harvesting Chlorophyll $a / b$ Complex (LHCII) Measured by EPR. J. Biol. Chem. 2012, 287, 2915-2925.

(5) Visser, H. M.; Kleima, F. J.; van Stokkum, I. H. M.; van Grondelle, R.; van Amerongen, H. Probing the Many Energy-Transfer Processes in the Photosynthetic Light-Harvesting Complex II at 77 K Using Energy-Selective SubPicosecond Transient Absorption Spectroscopy. Chem. Phys. 1996, 210, 297-312.

(6) Kleima, F. J.; Gradinaru, C. C.; Calkoen, F.; van Stokkum, I. H. M.; van Grondelle, R.; van Amerongen, H. Energy Transfer in LHCII Monomers at $77 \mathrm{~K}$ Studied by Sub-Picosecond Transient Absorption Spectroscopy. Biochemistry 1997, 36, 15262-15268. 
(7) Gradinaru, C. C.; Özdemir, S.; Gülen, D.; van Stokkum, I. H. M.; van Grondelle, R.; van Amerongen, H. The Flow of Excitation Energy in LHCII Monomers: Implications for the Structural Model of the Major Plant Antenna. Biophys. J. 1998, 75, 3064-3077.

(8) Bittner, T.; Wiederrecht, G. P.; Irrgang, K.-D.; Renger, G.; Wasielewski, M. R. Femtosecond Transient Absorption Spectroscopy on the Light-Harvesting Chl $a / b$ Protein Complex of Photosystem II at Room Temperature and 12 K. Chem. Phys. 1995, 194, 311-322.

(9) Kwa, S. L. S.; van Amerongen, H.; Lin, S.; Dekker, J. P.; van Grondelle, R.; Struve, W. S. Ultrafast EnergyTransfer in LHC-II trimers from the $\mathrm{Chl}$ a/b Light-Harvesting Antenna of Photosystem-II. Biochim. Biophys. Acta 1992, 1102, 202-212.

(10) Bittner, T.; Irrgang, K. D.; Renger, G.; Wasielewski, M. R. Ultrafast Excitation-Energy Transfer and ExcitonExciton Annihilation Processes in Isolated Light-Harvesting Complexes of Photosystem-II (LHC-II) from Spinach. $J$. Phys. Chem. 1994, 98, 11821-11826.

(11) Connelly, J. P.; Müller, M. G.; Hucke, M.; Gatzen, G.; Mullineaux, C. W.; Ruban, A. V.; Horton, P.; Holzwarth, A. R. Ultrafast Spectroscopy of Trimeric Light-Harvesting Complex II from Higher Plants. J. Phys. Chem. $B$ 1997, 101, 1902-1909.

(12) Du, M.; Xie, X.; Mets, L.; Fleming, G. R. Direct Observation of Ultrafast Energy-Transfer Processes in Light Harvesting Complex II. J. Phys. Chem. 1994, 98, 4736-4741.

(13) Salverda, J. M.; Vengris, M.; Krueger, B. P.; Scholes, G. D.; Czamoleski, A. R.; Novoderezhkin, V.; van Amerongen, H.; van Grondelle, R. Energy Transfer in Light-Harvesting Complexes LHCII and CP29 of Spinach Studied with Three Pulse Echo Peak Shift and Transient Grating. Biophys. J. 2003, 84, 450-465.

(14) Agarwal, R.; Krueger, B. P.; Scholes, G. D.; Yang, M.; Yom, J.; Mets, L.; Fleming, G. R. Ultrafast Energy Transfer in LHC-II Revealed by Three-Pulse Photon Echo Peak Shift Measurements. J. Phys. Chem. B 2000, 104, 2908-2918.

(15) Pålsson, L. O.; Spangfort, M. D.; Gulbinas, V.; Gillbro, T. Ultrafast Chlorophyll b- Chlorophyll a Excitation Energy Transfer in the Isolated Light Harvesting Complex, LHC II, of Green Plants. Implications for the Organisation of Chlorophylls. FEBS Lett. 1994, 339, 134-138.

(16) Linnanto, J.; Martiskainen, J.; Lehtovuori, V.; Ihalainen, J.; Kananavicius, R.; Barbato, R.; Korppi-Tommola, J. Excitation Energy Transfer in the LHC-II Trimer: a Model Based on the New 2.72 Angstrom Structure. Photosynth. Res. 2006, 87, 267-279.

(17) Schlau-Cohen, G. S.; Calhoun, T. R.; Ginsberg, N. S.; Read, E. L.; Ballottari, M.; Bassi, R.; van Grondelle, R.; Fleming, G. R. Pathways of Energy Flow in LHCII from Two-Dimensional Electronic Spectroscopy. J. Phys. Chem. B 2009, 113, 15352-15363.

(18) Novoderezhkin, V. I.; Palacios, M. A.; van Amerongen, H.; van Grondelle, R. Excitation Dynamics in the LHCII Complex of Higher Plants: Modeling Based on the 2.72 Angstrom Crystal Structure. J. Phys. Chem. B 2005, 109, 10493-10504.

(19) Liu, Z. F.; Yan, H. C.; Wang, K. B.; Kuang, T. Y.; Zhang, J. P.; Gui, L. L.; An, X. M.; Chang, W. R. Crystal Structure of Spinach Major Light-Harvesting Complex at 2.72 Angstrom Resolution. Nature 2004, 428, 287-292.

(20) Renger, T.; Schlodder, E. Primary Photophysical Processes in Photosystem II: Bridging the Gap between Crystal Structure and Optical Spectra. ChemPhysChem 2010, 11, 1141-1153.

(21) Renger, T.; Madjet, M. E.; Knorr, A.; Müh, F. How the Molecular Structure Determines the Flow of Excitation Energy in Plant Light-Harvesting Complex II. J. Plant Physiol 2011, 168, 1497-1509.

(22) Brixner, T.; Stenger, J.; Vaswani, H. M.; Cho, M.; Blankenship, R. E.; Fleming, G. R. Two-Dimensional Spectroscopy of Electronic Couplings in Photosynthesis. Nature 2005, 434, 625-628.

(23) Lewis, K. L.; Ogilvie, J. P. Probing Photosynthetic Energy and Charge Transfer with Two-Dimensional Electronic Spectroscopy. J. Phys. Chem. Lett. 2012, 3, 503-510. 
(24) Calhoun, T. R.; Ginsberg, N. S.; Schlau-Cohen, G. S.; Cheng, Y.-C.; Ballottari, M.; Bassi, R.; Fleming, G. R. Quantum Coherence Enabled Determination of the Energy Landscape in Light-Harvesting Complex II. J. Phys. Chem. $B$ 2009, 113, 16291-16295.

(25) Wells, K. L.; Lambrev, P. H.; Zhang, Z. Y.; Garab, G.; Tan, H. S. Pathways of Energy Transfer in LHCII Revealed by Room-Temperature 2D Electronic Spectroscopy. Phys. Chem. Chem. Phys. 2014, 16, 11640-11646.

(26) Enriquez, M. M.; Akhtar, P.; Zhang, C.; Garab, G.; Lambrev, P. H.; Tan, H. S. Energy Transfer Dynamics in Trimers and Aggregates of Light-Harvesting Complex II Probed by 2D Electronic Spectroscopy. J. Chem. Phys. 2015, $142,212432$.

(27) Zhang, Z.; Lambrev, P. H.; Wells, K. L.; Garab, G.; Tan, H. S. Direct Observation of Multistep Energy Transfer in LHCII with Fifth-Order 3D Electronic Spectroscopy. Nat. Commun. 2015, 6, 7914.

(28) Duan, H.-G.; Stevens, A. L.; Nalbach, P.; Thorwart, M.; Prokhorenko, V. I.; Miller, R. J. D. Two-Dimensional Electronic Spectroscopy of Light-Harvesting Complex II at Ambient Temperature: a Joint Experimental and Theoretical Study. J. Phys. Chem. B 2015, 119, 12017-12027.

(29) Fuller, F. D.; Ogilvie, J. P. Experimental Implementations of Two-Dimensional Fourier Transform Electronic Spectroscopy. Annu. Rev. Phys. Chem. 2015, 66, 667-690.

(30) Barzda, V.; Gulbinas, V.; Kananavicius, R.; Cervinskas, V.; van Amerongen, H.; van Grondelle, R.; Valkunas, L. Singlet-Singlet Annihilation Kinetics in Aggregates and Trimers of LHCII. Biophys. J. 2001, 80, 2409-2421.

(31) Zhang, Z.; Wells, K. L.; Hyland, E. W. J.; Tan, H.-S. Phase-Cycling Schemes for Pump-Probe Beam Geometry Two-Dimensional Electronic Spectroscopy. Chem. Phys. Lett. 2012, 550, 156-161.

(32) Myers, J. A.; Lewis, K. L.; Tekavec, P. F.; Ogilvie, J. P. Two-Color Two-Dimensional Fourier Transform Electronic Spectroscopy with a Pulse-Shaper. Opt. Express 2008, 16, 17420-17428.

(33) Palacios, M. A.; de Weerd, F. L.; Ihalainen, J. A.; van Grondelle, R.; van Amerongen, H. Superradiance and Exciton (De)Localization in Light-Harvesting Complex II from Green Plants? J. Phys. Chem. B 2002, 106, 57825787.

(34) Schubert, A.; Beenken, W. J. D.; Stiel, H.; Voigt, B.; Leupold, D.; Lokstein, H. Excitonic Coupling of Chlorophylls in the Plant Light-Harvesting Complex LHC-II. Biophys. J. 2002, 82, 1030-1039.

(35) Mullineaux, C. W.; Pascal, A. A.; Horton, P.; Holzwarth, A. R. Excitation-Energy Quenching in Aggregates of the LHC II Chlorophyll-Protein Complex: a Time-Resolved Fluorescence Study. Biochim. Biophys. Acta 1993, $1141,23-28$.

(36) Lambrev, P. H.; Schmitt, F. J.; Kussin, S.; Schoengen, M.; Várkonyi, Z.; Eichler, H. J.; Garab, G.; Renger, G. Functional Domain Size in Aggregates of Light-Harvesting Complex II and Thylakoid Membranes. Biochim. Biophys. Acta 2011, 1807, 1022-1031.

(37) Novoderezhkin, V. I.; van Grondelle, R. Physical Origins and Models of Energy Transfer in Photosynthetic Light-Harvesting. Phys. Chem. Chem. Phys. 2010, 12, 7352-7365.

(38) Novoderezhkin, V.; Marin, A.; van Grondelle, R. Intra- and Inter-Monomeric Transfers in the Light Harvesting LHCII Complex: the Redfield-Forster Picture. Phys. Chem. Chem. Phys. 2011, 13, 17093-17103.

(39) Caffarri, S.; Croce, R.; Breton, J.; Bassi, R. The Major Antenna Complex of Photosystem II Has a Xanthophyll Binding Site Not Involved in Light Harvesting. J. Biol. Chem. 2001, 276, 35924-35933.

(40) Spencer, A. P.; Li, H.; Cundiff, S. T.; Jonas, D. M. Pulse Propagation Effects in Optical 2D Fourier-Transform Spectroscopy: Theory. J. Phys. Chem. A 2015, 119, 3936-3960.

(41) Yan, S. X.; Tan, H. S. Phase Cycling Schemes for Two-Dimensional Optical Spectroscopy with a Pump-Probe Beam Geometry. Chem. Phys. 2009, 360, 110-115.

(42) Verluise, F.; Laude, V.; Cheng, Z.; Spielmann, C.; Tournois, P. Amplitude and Phase Control of Ultrashort Pulses by Use of an Acousto-Optic Programmable Dispersive Filter: Pulse Compression and Shaping. Opt. Lett. 2000, $25,575-577$. 